\title{
Correspondance
}

\section{Drug therapy for Alzheimer's disease}

W

e would like to highlight several shortcomings in the meta-analysis of cholinesterase inhibitors in Alzheimer's disease presented by Krista Lanctôt and associates $^{1}$ and to clarify information on the risk-benefit ratio for galantamine.

First, some of the data used in the meta-analysis were obtained with subtherapeutic or higher-than-recommended doses, and treatment duration varied widely (12 weeks to 1 year). Consequently, the heterogeneity reported by Lanctôt and associates ${ }^{1}$ is unsurprising. Focusing on data obtained with recommended maintenance doses for common treatment durations would yield more reliable and clinically relevant information.

Second, the use of global clinical measures for examining patient change is problematic because of the processes used to obtain these results (usually semistructured interviews): with this method, the domains covered and specific questions used may vary from one subject to another. Furthermore, the relative contribution of changes in different domains to the overall clinical judgement about change from baseline values varies between clinicians. Interrater reliability and differences between the 2 assessment scales used in the study by Lanctôt and associates are also of concern. ${ }^{2}$ Given the degenerative nature of Alzheimer's disease, limiting the definition of treatment success to improvement may be too restrictive. Rather, an overall positive response encompasses not only an increase in the number of patients showing improvement, but also a reduction in the number showing (greater) deterioration, especially over the longer term. For example, longer-term clinical trials of galantamine ( 5 to 6 months) have shown statistically significant benefit of 16-mg and 24-mg doses over placebo. ${ }^{3-5}$ More generally, given the importance of cognition to Alzheimer's disease and the relatively smaller inter-rater variation in this measure, we suggest that more weight be given to findings for the cognitive portion of the Alzheimer's Disease Assessment Scale (ADAS-Cog).

The data presented on the safety of galantamine do not reflect product labelling and recommended use in clinical settings. The clinical development of this drug has involved an evolution away from high doses (32 mg) and fast (weekly) titration $^{3,4,6,7}$ toward lower dosing (16 and 24 $\mathrm{mg}$ ) with a slower (monthly) titration schedule. ${ }^{5}$ By combining data from early and later trials, Lanctôt and associates ${ }^{1}$ determined discontinuation rates that incorrectly appear higher than those for donepezil or rivastigmine. Safety data from a trial in which dosing was consistent with product labelling ${ }^{5}$ showed discontinuation rates due to adverse events similar to placebo (odds ratio [OR] for $16-\mathrm{mg}$ dose $0.97,95 \%$ confidence interval [CI] 0.51 to 1.86 ; OR for 24-mg dose $1.46,95 \%$ CI 0.80 to 2.64$){ }^{8}$ The similarity in safety profiles of galantamine and donepezil was confirmed in a recent comparative trial. $^{9}$

\section{Shane Kavanagh}

Johnson \& Johnson Pharmaceutical

Services

Beerse, Belgium

\section{Patricia Kabathova}

Johnson \& Johnson Pharmaceutical

Services

Raritan, NJ

\section{References}

1. Lanctôt KL, Herrmann N, Yau KK, Khan LR, Liu BA, LouLou MM, et al. Efficacy and safety of cholinesterase inhibitors in Alzheimer's disease: a meta-analysis. CMA7 2003;169(6):557-64.

2. Schneider L. CIBIC+: What, why and how? Alzheimer Insights 1997; Jun:2-8.

3. Raskind MA, Peskind PR, Wessel T, Yuan W Galantamine in AD: a 6-month randomised, placebo-controlled trial with a 6-month extension. The Galantamine USA-1 Study Group. Neurology 2000;54:2261-8.

4. Wilcock G, Lilienfeld S, Gaens E. Efficacy and safety of galantamine in patients with mild to moderate Alzheimer's disease: a multi-centre randomised controlled trial. Galantamine International-1 Study Group. BMF 2000;321:1445-9.

5. Tariot PN, Solomon PR, Morris JC, Kershaw P A 5-month, randomised, placebo-controlled trial 
of galantamine in AD. The Galantamine USA10 Study Group. Neurology 2000;54:2269-76.

6. Wilkinson D, Murray J. Galantamine: a randomised, double-blind, dose comparison in patients with Alzheimer's disease. Int 7 Geriatr Psychiatry 2001;16:852-7.

7. Rockwood K, Mintzer J, Truyen L, Wessel T, Wilkinson D. Effects of a flexible galantamine dose in Alzheimer's disease: a randomised, controlled trial. 7 Neurol Neurosurg Psychiatry 2001, 71:589-95

8. Olin J, Schneider L. Galantamine for Alzheimer's disease [Cochrane review]. In: The Cochrane Library; Issue 4, 2001. Oxford: Update Software.

9. Wilcock G, Howe I, Coles H, Lilienfeld S, Truyen L, Zhu Y, et al. A long-term comparison of galantamine and donepezil in the treatment of Alzheimer's disease. Drugs Aging 2003;20:777-89.

Competing interests: The authors are employed within Johnson \& Johnson Pharmaceutical Services. Reminyl, a brand of galantamine, was developed by the research and development arm of Johnson \& Johnson and is marketed around the world by various operating companies of Johnson \& Johnson.

DOI:10.1053/cmaj.1031912

$\mathrm{A}^{\mathrm{lt}}$ though generally interesting, the meta-analysis by Krista Lanctôt and associates $^{1}$ of the efficacy and safety of cholinesterase inhibitors in the treatment of Alzheimer's disease simply demonstrates what is observed in day-to-day clinical practice: a slight improvement or stabilization of the disease (as indicated by neuropsychological evaluation) and various gastrointestinal side effects. The meta-analysis showed a slightly greater benefit with donepezil than with the other drugs - again, just what is observed in daily clinical practice.

Unfortunately, the extensive support that some of the authors received from the pharmaceutical industry, as outlined in the competing interests statement, ${ }^{1}$ might taint readers' perception of the authors' autonomy and independent interpretation of their findings. It is of course important to disclose potential competing interests, yet it is very difficult for readers to evaluate their influence on a study's conclusions. Furthermore, it might be useful if meta-analyses were to also include the competing interests of the authors of the papers included in the analysis. For example, the Japanese study ${ }^{2}$ that showed a substantial treatment effect with donepezil - ascribed in the meta-analysis ${ }^{1}$ to specific gene frequencies in the Japanese population - might have been influenced by the competing interests of its authors. As more and more clinical research is funded by companies with a financial interest in the outcomes, there may be greater pressure on researchers to assess and report their financial interests.

\section{Celio Levyman}

Neurologist

Saó Paulo, Brazil

\section{References}

1. Lanctôt KL, Herrmann N, Yau KK, Khan LR, Liu BA, LouLou MM, et al. Efficacy and safety of cholinesterase inhibitors in Alzheimer's disease: a meta-analysis. CMA7 2003;169(6):557-64.

2. Homma A, Takeda M, Imai Y, Udaka F, Hasegawa K, Kameyama M, et al. Clinical efficacy and safety of donepezil on cognitive and global function in patients with Alzheimer's disease. A 24-week, multicenter, double-blind, placebo-controlled study in Japan. E2020 Study Group. Dement Geriatr Cogn Disord 2000;11: 299-313.

Competing interests: None declared.

DOI:10.1053/cmaj.1031685

\section{[Three of the authors respond:]}

$\mathrm{T}$ he authors of these 2 letters raise several issues regarding our metaanalysis of the pivotal trials for 3 cholinesterase inhibitors used in the treatment of Alzheimer's disease.

Shane Kavanagh and Patricia Kabathova argue that the meta-analysis treats galantamine unfairly. In support of this claim, they point out that the majority of the galantamine trials included in the analysis did not use recommended doses. We explicitly recognized this and adjusted the meta-analysis of efficacy accordingly. They also suggest that metaanalyses focus on common treatment durations. However, there is no such thing as a "common treatment duration" in this context. In fact, the range of treatment duration is even greater than 12 weeks to 1 year, which Kavanagh and Kabathova refer to as "wide variation." We chose to be explicit about the treatment durations that were included and handled the differences by means of a subanalysis, assessing heterogeneity and providing confidence intervals.

In addition, Kavanagh and $\mathrm{Ka}-$ bathova suggest that more weight be given to the ADAS-cog assessment than to global clinical judgement. In keeping with the approach of health regulatory agencies such as the US Food and Drug
Administration, ${ }^{2}$ we provided the numbers needed to treat (NNT) and confidence intervals for both cognitive outcomes (the ADAS-cog scores) and global clinical impression. The emphasis on global outcomes was consistent with the stated goal of the analysis.

Kavanagh and Kabathova, Celio Levyman and other authors, ${ }^{3}$ all comment on the issue of comparability between the 3 drugs. However, in our meta-analysis we cautioned that individual cholinesterase inhibitors cannot be directly compared until welldesigned, properly blinded, head-tohead trials are conducted. Although Levyman shares his own clinical experience of a slightly greater benefit with donepezil, Kavanagh and Kabathova cite new evidence supporting the similarity in safety profiles between galantamine and other cholinesterase inhibitors. The study they selected as an example of a head-to-head trial ${ }^{4}$ was 1 of 3 published to date and was not available when we prepared our meta-analysis. All 3 studies $^{4-6}$ had relatively small numbers of subjects, and none was completely double-blinded. In each case, the results presented favoured the sponsor's drug. The similarities or differences among various cholinesterase inhibitors in terms of efficacy and safety remain to be established.

Levyman rightly points out that while the competing interests of the authors of the meta-analysis were fully disclosed, the primary randomized controlled trials used in the meta-analysis might themselves have been subject to bias. The bias we fear is the presentation of results unreasonably or unjustifiably favouring those of the sponsor or disfavouring those of a competitor. Although we cannot control for such bias, we did obtain and present results that were not distorted from what is found in clinical practice, that did not unduly favour the drug of any given sponsor and that did not unduly favour those drugs as a group or class. Bias also influences which trials are published, with negative trials less likely to be published. ${ }^{5}$ We addressed this potential problem by using funnel plots.

Meta-analysts and, more important, clinicians practising evidence-based 